\title{
Efecto de la variación del sustrato en la productividad de dos cepas de Pleurotus spp.
}

\section{Effect of substrate variation on the productivity of two strains of Pleurotus spp.}

\author{
Dora Onely Roblero-Mejía 1, Liliana Aguilar-Marcelino 2, José E. Sánchez ${ }^{1}$
}

${ }^{1}$ El Colegio de la Frontera Sur. Carretera al antiguo aeropuerto km 2.5, C.P. 30700, Tapachula, Chiapas, México.

${ }^{2}$ Centro Nacional de Investigación Disciplinaria en Salud Animal e Inocuidad, INIFAP. km 11 carretera federal Cuernavaca-Cuautla No. 8534, Col. Progreso, C.P. 62550, Jiutepec, Morelos, México.

\section{RESUMEN}

Antecedentes: En los últimos años se ha presentado un marcado interés por la producción comercial de Pleurotus spp., lo cual ha ocasionado la búsqueda de sustratos o mezclas que aseguren una alta productividad.

Objetivos: Determinar el efecto de los principales componentes químicos del sustrato en la producción de basidiomas de dos cepas del género Pleurotus.

Métodos: A partir de olote, pasto y pulpa de café se prepararon 10 tratamientos, que fueron analizados bromatológicamente. Se cultivaron dos cepas de Pleurotus spp. y se evaluó la eficiencia biológica (EB), la tasa de producción (TP) y el rendimiento $(\mathrm{R})$.

Resultados y conclusiones: En ambas cepas cultivadas, ECS-123 y ECS-1123, el tratamiento 7 (100 \% pasto) obtuvo los valores más altos de EB (38.2 \% y $48.8 \%$, respectivamente), TP (0.5\% y $0.6 \%$, respectivamente) y R (14.7 y $18.8 \%$, respectivamente). Estos valores no fueron estadísticamente diferentes de otros tratamientos con pasto, pero sí de la pulpa de café (con mayor contenido de nitrógeno). Se concluye que es suficiente una concentración de nitrógeno de $0.5 \%$ y una relación $\mathrm{C} / \mathrm{N}$ de 96.75 para obtener una buena producción de basidiomas. Se resalta la incidencia de otros factores en la productividad de las cepas.

Palabras clave: residuos lignocelulósicos, cuerpos fructíferos, Pleurotus ostreatus, P. djamor, eficiencia biológica

\section{ABSTRACT}

Background: Pleurotus is one of the best-known edible mushroom genera. In recent years there has been a marked interest in its commercial production, which has led to the search for substrates or mixtures that ensure high productivity. Objective: To determine the effect of the main chemical components of substrates for the production of two strains of Pleurotus spp.

Methods: Different mixtures were made from corn cob, grass and coffee pulp, establishing 10 treatments, which were bromatologically analyzed. Two strains of Pleurotus spp. were cultivated. The Biological Efficiency (BE), Production Rate (TP) and Yield (R) were evaluated.

Results and conclusions: In both strains ECS-123 and ECS-1123, treatment 7 (100\% grass) obtained the highest values of EB ( $38.2 \%$ and $48.8 \%$, respectively), TP $(0.5 \%$ and $0.6 \%$, respectively) and R ( 14.7 and $18.8 \%$, respectively). These values were not statistically different from other treatments containing grass, but from the one with higher nitrogen content (coffee pulp) so it is stated that a nitrogen concentration of $0.5 \%$ and $\mathrm{C} / \mathrm{N}$ ratio of 96.75 are sufficient to obtain a good production of basidiomata. The importance of other factors on the production of strains is highlighted.

Keywords: lignocellulosic residues, fruiting bodies, Pleurotus ostreatus, P. djamor, biological efficiency

\section{ARTICLE HISTORY}

Received 16 October 2020 / Accepted 16 July 2021

On line 17 November 2021

\section{CORRESPONDING AUTHOR}

\usé E. Sánchez, esanchez@ecosur.mx

ORCID 0000-0003-0535-4302 


\section{INTRODUCCIÓN}

Los hongos comestibles son conocidos como productos alimenticios ricos en proteínas, minerales y componentes bioactivos, también destacan por sus propiedades medicinales que incluyen propiedades anticancerígenas, antibióticas, antivirales, antiinflamatorias, efecto inmunomodulador, efecto hipolipemiante, entre otros (Wani et al. 2010; Chang y Wasser 2018). Debido a su importancia, la producción mundial de hongos comestibles ha aumentado en las últimas décadas, en 1997 la producción mundial fue de 3 millones de toneladas, para 2007, esta cantidad aumentó casi el doble y para 2017 se registró una producción de 10 millones de toneladas (FAO 2017).

En la naturaleza, existe una gran cantidad de hongos comestibles (Gupta et al. 2018; Ruan-Soto 2018). Pleurotus es uno de los géneros más conocidos y ocupa el segundo lugar entre los hongos más consumidos a nivel mundial, después del shiitake (Royse et al. 2017). El género Pleurotus se caracteriza por su capacidad para degradar lignina selectivamente, sacarificar e hidrolizar celulosa y aumentar la digestibilidad de los sustratos para producir cuerpos fructíferos (Sharma et al. 2013). Esta capacidad para adaptar rápidamente su metabolismo a diversos sustratos depende de la habilidad para la formación de micelio y permitir su desarrollo, atribuida a su capacidad de secretar enzimas degradadoras como celulasas, hemicelulasas, xilanasas y ligninasas, las cuales varían en las especies de Pleurotus, de acuerdo con la cepa y tipo de sustrato, durante sus distintos estados de desarrollo (Periasamy y Natarajan 2004; Motato y Mejía 2006).

En los últimos años, alrededor del mundo, se ha presentado un marcado interés por la producción comercial de este género (Sánchez et al. 2008), favorecido por las diversas ventajas que presenta, entre ellas, la posibilidad de cultivarlo en zonas tropicales y de producir alimentos de alto valor nutrimental, utilizando una amplia gama de residuos agrícolas como sustrato que permite impulsar el abasto alimentario, la generación de ingresos económicos y el cuidado del ambiente (Gaitán-Hernández y Silva-Huerta 2016; Romero-Arenas et al. 2018). Teniendo en cuenta su interés comercial y biotecnológico, se busca el abaratamiento de los costos de producción mediante el aprovechamiento de residuos lignocelulósicos disponibles en cada región, tomando en cuenta la calidad y el éxito de la productividad del cultivo (Abid et al. 2020). La identificación de los residuos lignocelulósicos como sustrato adecuado, tanto biológica como económicamente, es fundamental para el cultivo exitoso (Bitew y Mandefro 2018).

El uso de diferentes sustratos para el cultivo de las especies de Pleurotus influye en los parámetros de productividad y composición química, lo que ha ocasionado un estudio intenso para obtener sustratos o mezclas que produzcan mayores eficiencias biológicas y de alta calidad (Piña-Guzmán et al. 2017). Las diferencias de productividad entre distintos sustratos son atribuibles a su composición química, física y biológica; disponibilidad de nutrientes, área biogeográfica, retención de la humedad, aspectos epigenéticos, entre otros factores (Girmay et al. 2016).

Debido a lo anterior, la comprensión del efecto que causan los principales nutrientes en la productividad y la búsqueda de sustratos o mezclas que aseguren altos rendimientos con cuerpos fructíferos de buena calidad, es una prioridad. Por ello, en este trabajo, al evaluar diferentes residuos agroindustriales y la combinación de ellos como sustratos para el cultivo de dos cepas de Pleurotus spp., se buscó determinar el efecto y la importancia de los principales componentes químicos en la producción de las cepas cultivadas.

\section{MATERIALES Y MÉTODOS}

\section{Material biológico}

Se emplearon las cepas Pleurotus ostreatus ECS-1123, de interés comercial, y Pleurotus djamor ECS-123, de interés biotecnológico debido a los metabolitos y enzimas que produce (Moreno-Ruiz et al. 2014; Cruz-Ornelas et al. 2019), ambas cepas están resguardadas en el cepario micológico de El Colegio de la Frontera Sur (ECOSUR) en Tapachula, Chiapas, México.

\section{Obtención del inóculo}

Para la preparación del inóculo, se emplearon semillas de sorgo lavadas, la humedad de las semillas se ajustó entre 60-70 \%, siguiendo las indicaciones de Quimio (2002). Posteriormente, el material se esterilizó en autoclave a $1.05 \mathrm{~kg} / \mathrm{cm} 2$ de presión, $121^{\circ} \mathrm{C}$ por $30 \mathrm{~min}$. Las semillas estériles y a temperatura ambiente, fueron inoculadas con micelio de las cepas de Pleurotus spp. ECS-123 y ECS-1123. Los inóculos se incubaron a $25^{\circ} \mathrm{C}$ en oscuridad por aproximadamente 20 días. 


\section{Sustratos}

Los materiales evaluados fueron tres residuos agroindustriales:1) pasto pangola, 2) pulpa de café y 3) olote de maíz. La pulpa de café fue obtenida como subproducto del beneficiado seco de café robusta Coffea canephora, una mezcla heterogénea de pulpa y cascarilla del grano de café. El pasto y el olote fueron obtenidos en el área agrícola de las inmediaciones de Tapachula, Chiapas, México y fueron fragmentados en partículas de 2-3 cm de longitud, con la ayuda de un molino eléctrico de disco de $7 \mathrm{HP}$.

\section{Preparación de los tratamientos}

A partir de los sustratos mencionados, se obtuvieron 10 tratamientos, siete fueron mezclas y tres fueron los residuos agroindustriales de manera individual (Tabla 1). Cada tratamiento consistió en $385 \mathrm{~g}$ de material seco (gramos de material seco necesarios para obtener un peso final de aproximadamente $1 \mathrm{~kg}$ en cada bolsa de tratamiento, con $60 \%$ de humedad), adicionado con cal apagada (hidróxido de calcio $0.5 \%$ ). Todos los tratamientos se colocaron en bolsas de polipapel de 20 x $35 \mathrm{~cm}$ y fueron esterilizados en autoclave a $1.05 \mathrm{~kg} /$ $\mathrm{cm}^{2}$ de presión, $121^{\circ} \mathrm{C}$ por $40 \mathrm{~min}$. Posteriormente se enfriaron a temperatura ambiente y se inocularon con $40 \mathrm{~g}$ de semillas de sorgo colonizadas con micelio de las cepas antes mencionadas. Todos los tratamientos se realizaron por triplicado.

Tabla 1. Composición de las mezclas de sustrato (tratamientos) utilizados para la producción de basidiomas de las cepas de Pleurotus spp.

\begin{tabular}{ccccc}
\hline Tratamiento & Olote & Pasto & Pulpa & Total (MS) \\
\hline $\mathrm{T}_{1}$ & 0.17 & 0.67 & 0.17 & 1.00 \\
$\mathrm{~T}_{2}$ & 0.17 & 0.17 & 0.67 & 1.00 \\
$\mathrm{~T}_{3}$ & 0 & 0.5 & 0.5 & 1.00 \\
$\mathrm{~T}_{4}$ & 0.67 & 0.17 & 0.17 & 1.00 \\
$\mathrm{~T}_{5}$ & 0.33 & 0.33 & 0.33 & 1.00 \\
$\mathrm{~T}_{6}$ & 0 & 0 & 1 & 1.00 \\
$\mathrm{~T}_{7}$ & 0 & 1 & 0 & 1.00 \\
$\mathrm{~T}_{8}$ & 1 & 0 & 0 & 1.00 \\
$\mathrm{~T}_{9}$ & 0.5 & 0.5 & 0 & 1.00 \\
$\mathrm{~T}_{10}$ & 0.5 & 0 & 0.5 & 1.00 \\
\hline
\end{tabular}

\section{Análisis bromatológico de los tratamientos}

Para conocer la composición química de los diferentes tratamientos (sustratos), los materiales fueron molidos hasta obtener un polvo fino, y fueron enviados al La- boratorio de Bromatología de ECOSUR, en donde se realizaron las siguientes determinaciones, siguiendo los lineamientos de la AOAC (Williams 1984): humedad y cenizas por el método gravimétrico; proteína por digestión $100 \mathrm{~min} / 398^{\circ} \mathrm{C}$, destilación con ácido bórico al 1 \% y mezcla de catalizadores, titulación con solución de ácido sulfúrico $0.0501 \mathrm{~N}$; nitrógeno por método de Kjeldahl; grasa por el método Soxhlet; fibra cruda por digestión ácida/alcalina; azúcares reductores por DNS, los carbohidratos totales se determinaron mediante la fórmula: \% carbohidratos $=100-(\%$ humedad $+\%$ cenizas $+\%$ proteína $+\%$ grasa $+\%$ fibra cruda). El porcentaje de carbono fue obtenido por la fórmula de Adams et al. (1951): \% Carbono = (\% VS) / 1.8, donde $\%$ VS $=100-\%$ cenizas.

\section{Incubación y fructificación}

Los tratamientos una vez inoculados, fueron incubados a $25^{\circ} \mathrm{C}$ en oscuridad por 3-5 semanas, según colonizaba cada tratamiento. Una vez que los tratamientos estuvieron completamente colonizados, fueron trasladadas al cuarto de fructificación en condiciones apropiadas de humedad $(80-90 \%)$, temperatura $\left(22-26^{\circ} \mathrm{C}\right)$, luz natural y aeración constante (Tshinyangu y Hennebert 1995).

\section{Parámetro de crecimiento de los cuerpos fructíferos}

Para la evaluación de productividad de las cepas en los diferentes sustratos se contaron y pesaron los hongos frescos de la primera y segunda cosecha por cada bolsa de tratamiento, a partir de estos datos se calculó la eficiencia biológica (EB), la tasa de producción (TP) y rendimiento $(R)$, mediante las siguientes fórmulas (Del Pilar Ríos et al. 2010):
$\mathrm{EB}(\%)=($ peso de los cuerpos fructíferos frescos $(\mathrm{g}) /$ peso del sustrato seco (g)) $\times 100$.

TP $(\%)=$ EB / tiempo transcurrido desde la inoculación del micelio en el sustrato hasta la última cosecha de hongos (número de días).

$$
\begin{gathered}
R=\text { (peso seco de los cuerpos fructíferos }(\mathrm{g}) / \text { peso } \\
\text { seco del sustrato }(\mathrm{g})) \times 100 .
\end{gathered}
$$

El secado de los hongos y de los sustratos se llevó a cabo en una cámara de deshidratación a $50{ }^{\circ} \mathrm{C}$ por tres días. 


\section{Análisis estadístico}

Se utilizó un diseño estadístico de dietas $A B C D$ de 10 tratamientos con tres repeticiones para cada cepa. Los datos obtenidos fueron sometidos a un análisis de varianza (ANOVA), y a una prueba de comparación de medias por el método de Tukey, con $95 \%$ de confiabilidad ( $\alpha=0.05)$, con el paquete estadístico JMP versión 7.0.1 (SAS, 2007).

\section{RESULTADOS}

\section{Composición bromatológica de los sustratos}

En las Tablas 2 y 3 se muestran los resultados del análisis bromatológico de los tratamientos evaluados como sustratos. Se obtuvieron valores de proteína entre 2.54 a $8.21 \%$ y de nitrógeno entre 0.41 a $1.31 \%$. El $\mathrm{T}_{6^{\prime}}$ que corresponde a $100 \%$ pulpa de café, obtuvo los porcentajes más altos de nitrógeno y de proteína, y en cuanto a la relación $\mathrm{C} / \mathrm{N}$ presentó el valor más bajo en comparación a los demás tratamientos, que fue de 39.79. Por otro lado, los tratamientos $T_{8}$ y $T_{9}$ presentaron los valores más bajos de nitrógeno, que fueron de 0.41 y $0.47 \%$, respectivamente, aunque presentaron las relaciones $\mathrm{C} / \mathrm{N}$ más altas, 131.41 y 111.87 , respectivamente; $T_{8}$ corresponde a $100 \%$ olote de maíz, y $T_{9}$ corresponde a la relación 1:1 de olote y pasto. El $T_{8}$ fue el tratamiento con mayor contenido de carbohidratos y azúcares reductores, mientras que el $\mathrm{T}_{6}$ obtuvo los valores más bajos en dichos parámetros. Los valores de grasa oscilaron entre 0.2-1.04 \% y los valores de ceniza entre $3.01-7.7 \%$.

Tabla 2. Composición bromatológica de los diferentes tratamientos utilizados para la producción de basidiomas de Pleurotus spp.

\begin{tabular}{|c|c|c|c|c|c|c|c|}
\hline Tratamientos & Humedad (\%) & Proteína & Fibra cruda & Grasa & Carbohidratos & $\begin{array}{l}\text { Azúcares reduc- } \\
\text { tores }\end{array}$ & Cenizas \\
\hline $\mathrm{T}_{1}$ & 8.63 & 4.05 & 39.29 & 0.69 & 40.62 & 20.81 & 6.72 \\
\hline $\mathrm{T}_{2}$ & 8.76 & 6.50 & 38.85 & 0.85 & 39.09 & 16.84 & 5.95 \\
\hline $\mathrm{T}_{3}$ & 9.80 & 5.77 & 38.45 & 0.88 & 38.17 & 17.74 & 6.94 \\
\hline $\mathrm{T}_{4}$ & 8.09 & 3.66 & 39.77 & 0.43 & 43.68 & 21.50 & 4.38 \\
\hline $\mathrm{T}_{5}$ & 10.31 & 4.64 & 38.52 & 0.64 & 40.31 & 19.32 & 5.57 \\
\hline $\mathrm{T}_{6}$ & 9.93 & 8.21 & 38.01 & 1.04 & 36.64 & 13.76 & 6.17 \\
\hline$T_{7}$ & 9.68 & 3.32 & 38.89 & 0.71 & 39.70 & 21.71 & 7.70 \\
\hline$T_{8}$ & 8.59 & 2.54 & 39.84 & 0.20 & 45.82 & 23.09 & 3.01 \\
\hline$T_{9}$ & 9.13 & 2.93 & 39.37 & 0.46 & 42.76 & 22.40 & 5.36 \\
\hline$T_{10}$ & 9.26 & 5.38 & 38.93 & 0.62 & 41.23 & 18.43 & 4.59 \\
\hline
\end{tabular}

El cálculo de los valores se refiere a gramos por cada $100 \mathrm{~g}$ de materia seca.

Tabla 3. Contenido de carbono $(\mathrm{C})$, nitrógeno $(\mathrm{N})$ y relación carbono/nitrógeno $(\mathrm{C} / \mathrm{N})$ de los diferentes tratamientos utilizados para la producción de basidiomas de Pleurotus spp.

\begin{tabular}{cccc}
\hline Tratamientos & $\mathrm{C}(\%)$ & $\mathrm{N}(\%)$ & $\mathrm{C} / \mathrm{N}$ \\
\hline $\mathrm{T}_{1}$ & 51.82 & 0.65 & 79.72 \\
$\mathrm{~T}_{2}$ & 52.25 & 1.04 & 50.24 \\
$\mathrm{~T}_{3}$ & 51.70 & 0.92 & 56.19 \\
$\mathrm{~T}_{4}$ & 53.12 & 0.59 & 90.03 \\
$\mathrm{~T}_{5}$ & 52.46 & 0.74 & 70.89 \\
$\mathrm{~T}_{6}$ & 52.13 & 1.31 & 39.79 \\
$\mathrm{~T}_{7}$ & 51.28 & 0.53 & 96.75 \\
$\mathrm{~T}_{8}$ & 53.88 & 0.41 & 131.41 \\
$\mathrm{~T}_{9}$ & 52.58 & 0.47 & 111.87 \\
$\mathrm{~T}_{10}$ & 53.01 & 0.86 & 61.63 \\
\hline
\end{tabular}


Parámetros de producción y productividad

Eficiencia biológica. Los valores de EB de las cepas de Pleurotus spp. obtenidos con las diferentes mezclas y sustratos evaluados se muestran en la Figura 1.

Los valores obtenidos de las EBs correspondientes a la cepa $P$. djamor ECS-123 se clasificaron en tres grupos estadísticos: A, B y C. El T (100\% pasto) fue el sustrato en que esta cepa produjo la mayor $E B(E B=38.2 \%)$, siendo estadísticamente diferente sólo de $T_{6}(100 \%$ pulpa de café) y $\mathrm{T}_{10}$ (1:1 olote y pulpa de café), ya que se obtuvieron los valores más bajos (EBs de $20.8 \%$ y $20.2 \%$, respectivamente).

En cuanto a la cepa $P$. ostreatus ECS-1123, el análisis estadístico separó los valores promedio de EB en dos grupos diferentes: a y b. El valor más alto para esta cepa se obtuvo en el $\mathrm{T}_{7}$ (100 \% pasto) con una EB de $48.8 \%$, este valor fue estadísticamente similar a las EBs de siete de los tratamientos probados: $\mathrm{T}_{1}, \mathrm{~T}_{2^{\prime}}, \mathrm{T}_{3^{\prime}}, \mathrm{T}_{4^{\prime}} \mathrm{T}_{5^{\prime}}, \mathrm{T}_{9} \mathrm{y} \mathrm{T}_{10^{\prime}}$ pero diferente de $\mathrm{T}_{6}$ (100\% pulpa de café) y $\mathrm{T}_{8}(100 \%$ olote), los cuales obtuvieron los valores de EB más bajos (16.9 y $24.6 \%$, respectivamente), estos dos tratamientos no tuvieron diferencias significativas entre sí.

El efecto de cada sustrato en la EB es presentado en la Figura 2, para ambas cepas es notorio que, a mayor cantidad de pasto en los tratamientos, las EBs se incrementan. Mientras que, a mayor cantidad de pulpa de café en los tratamientos, las EBs disminuyen.
En el caso del olote de maíz, los resultados variaron dependiendo de la cepa, ya que las muestras inoculadas con $P$. djamor ECS-123 presentaron menores porcentajes de EB cuando se aumentó el contenido de olote, mientras que para $P$. ostreatus ECS-1123 los mejores EBs se alcanzaron cuando el tratamiento contenía una fracción de 0.33 del sustrato, disminuyendo su productividad a mayores contenidos del olote de maíz.

Peso promedio, productividad y rendimiento. En la Tabla 4 se muestran los resultados de los parámetros de productividad de las cepas evaluadas bajo los diferentes tratamientos. El peso promedio de los hongos cosechados con ambas cepas fue mayor en el $\mathrm{T}_{7}$, siendo estadísticamente diferente sólo de $\mathrm{T}_{6}$ y $\mathrm{T}_{10}$ en la cepa ECS-123, y de $\mathrm{T}_{6}$ y $\mathrm{T}_{8}$ en la cepa ECS-1123. El mismo comportamiento se observó para la tasa de producción (TP) y rendimiento $(R)$, con valores de 0.1 hasta $0.6 \%$ y de 6.5 a $18.8 \%$, respectivamente. Por otro lado, el número de hongos cosechados en los diferentes tratamientos de la cepa ECS-123 no presentaron diferencias significativas, con valores que oscilaron entre 30.2 a 62.8 hongos por bolsa. Para el caso de la cepa ECS-1123, $T_{7}$ presentó el número más alto de cuerpos fructíferos cosechados por bolsa de 46.2, este valor fue estadísticamente similar a los tratamientos $T_{1}$,

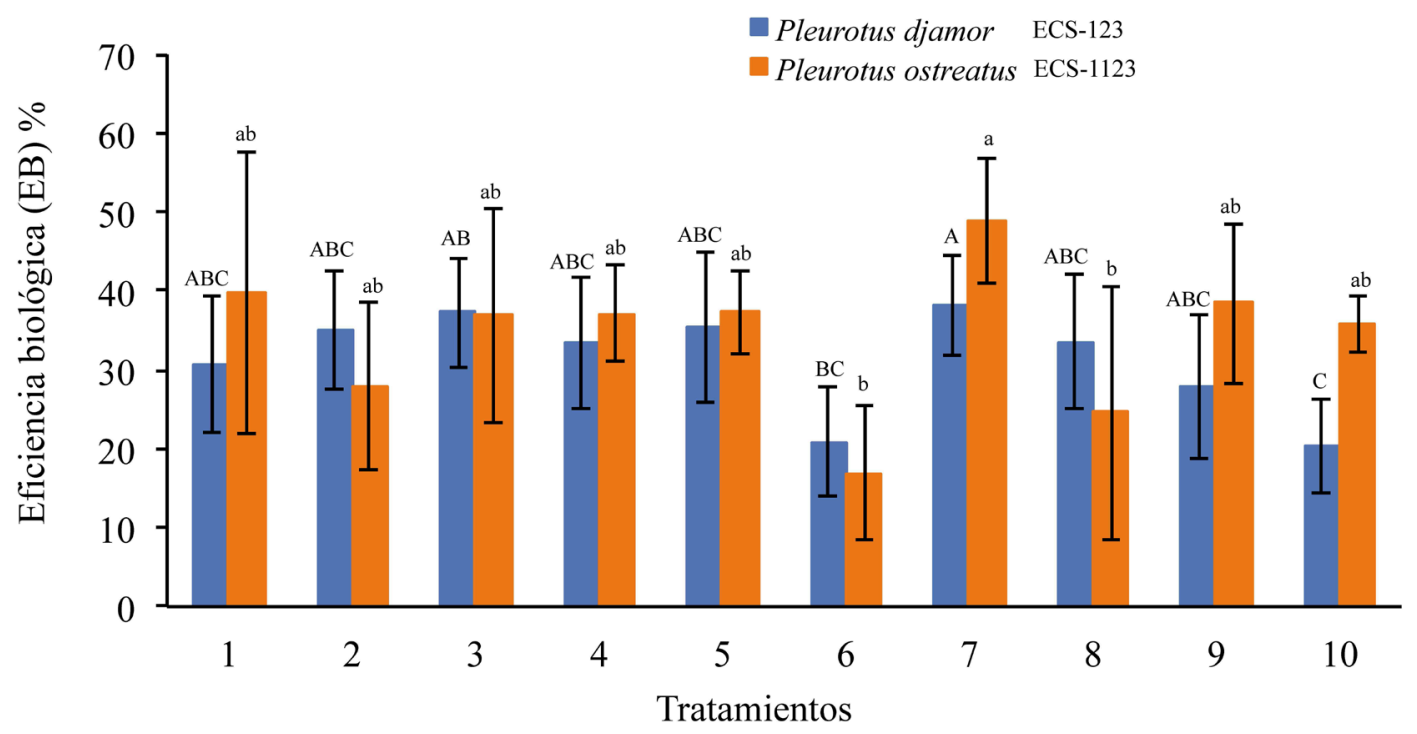

Figura 1. Efecto de la variación del sustrato en la productividad de dos cepas de Pleurotus spp. La composición de los tratamientos se menciona en la Tabla 1. Las líneas sobre las barras representan la desviación estándar de tres repeticiones. Letras iguales indican que no hay diferencias significativas (en letras mayúsculas para el análisis de los tratamientos de la cepa ECS-123 y en minúscula para la cepa ECS-1123, a través de la prueba Tukey $\alpha=0.05)$. 

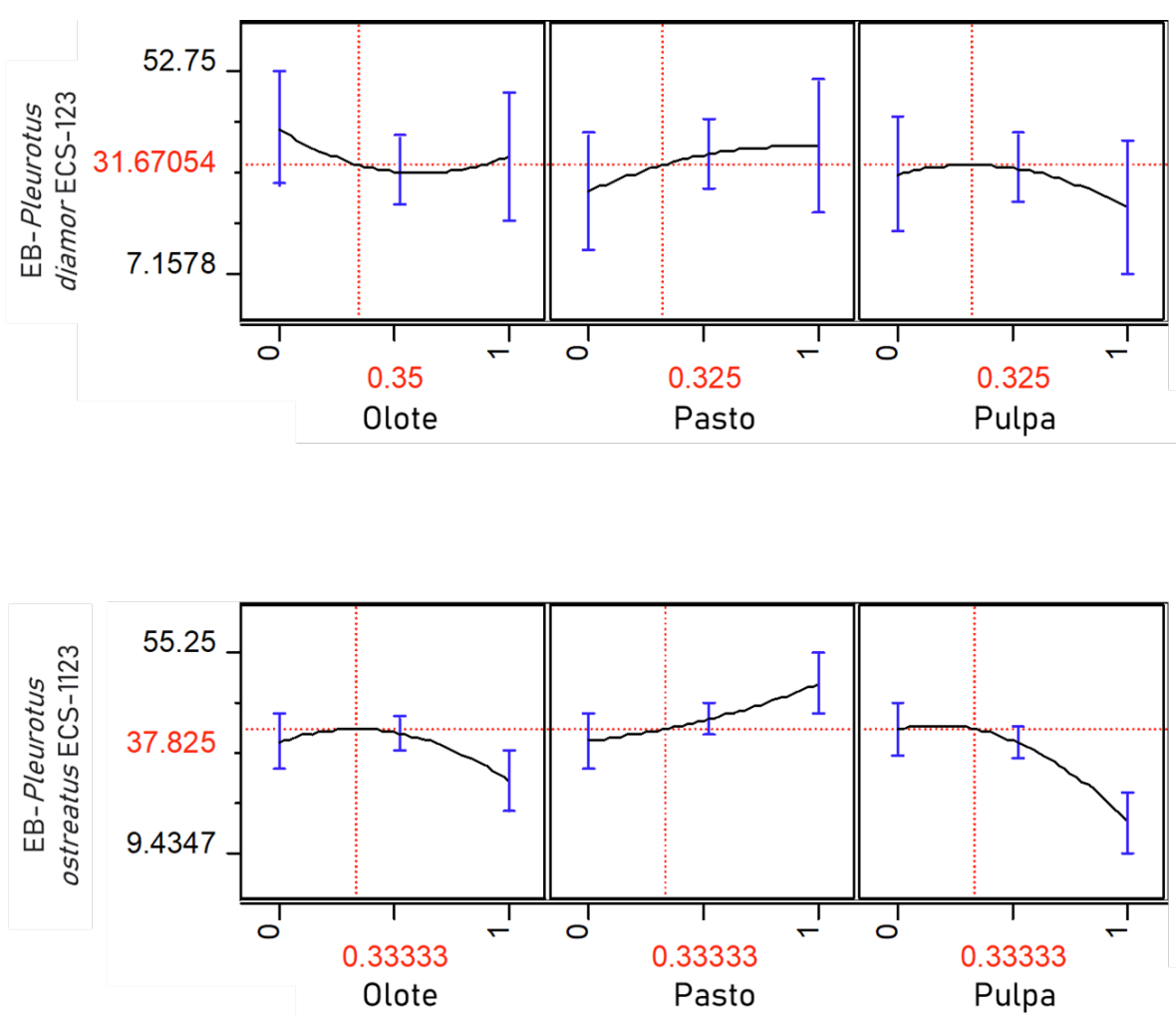

Figura 2. Efecto del sustrato (olote, pasto y pulpa de café) sobre la EB de las cepas cultivadas. Las líneas punteadas horizontales hacen referencia a la media de respuesta de la EB y las líneas punteadas verticales hacen referencia al intercepto de la media de respuesta de la EB con el eje $y$.

Tabla 4. Producción de basidiomas de P. djamor ECS-123 y P. ostreatus ECS-1123 cultivadas en cada uno de los tratamientos evaluados

\begin{tabular}{|c|c|c|c|c|c|c|c|c|}
\hline & \multicolumn{2}{|c|}{$\begin{array}{l}\text { Peso promedio de hongos } \\
\text { cosechados por bolsa (g) }\end{array}$} & \multicolumn{2}{|c|}{$\begin{array}{l}N^{\circ} \text { promedio de hongos } \\
\text { cosechados por bolsa }\end{array}$} & \multicolumn{2}{|c|}{ Tasa de producción (\%) } & \multicolumn{2}{|c|}{ Rendimiento (\%) } \\
\hline & ECS-123 & ECS-1123 & ECS-123 & ECS-1123 & ECS-123 & ECS-1123 & ECS-123 & ECS-1123 \\
\hline 1 & $118.4 \pm 33.3 \mathrm{abc}$ & $152.8 \pm 68.3 a b$ & $42.4 \pm 15.7 a$ & $40.2 \pm 17.7 \mathrm{ab}$ & $0.4 \pm 0.1 \mathrm{abc}$ & $0.4 \pm 0.2 \mathrm{ab}$ & $11.8 \pm 3.3 a$ & $15.2 \pm 6.8 \mathrm{ab}$ \\
\hline 2 & $134.9 \pm 28.7 \mathrm{abc}$ & $107.8 \pm 41.0 \mathrm{ab}$ & $52.2 \pm 15.4 a$ & $32.5 \pm 12.2 \mathrm{ab}$ & $0.4 \pm 0.1 \mathrm{ab}$ & $0.3 \pm 0.1 b c$ & $11.5 \pm 6.3 a$ & $10.7 \pm 4.1 \mathrm{ab}$ \\
\hline 3 & $143.6 \pm 26.7 \mathrm{ab}$ & $142.2 \pm 52.5 \mathrm{ab}$ & $59.6 \pm 36.8 a$ & $38.8 \pm 24.4 \mathrm{ab}$ & $0.5 \pm 0.1 a$ & $0.4 \pm 0.1 \mathrm{ab}$ & $14.3 \pm 2.7 a$ & $14.2 \pm 5.2 \mathrm{ab}$ \\
\hline 4 & $128.6 \pm 32 \mathrm{abc}$ & $143.2 \pm 23.3 \mathrm{ab}$ & $62.8 \pm 36.7 a$ & $35.2 \pm 9.8 \mathrm{ab}$ & $0.4 \pm 0.1 \mathrm{a}$ & $0.4 \pm 0.1 \mathrm{ab}$ & $12.8 \pm 3.2 \mathrm{a}$ & $14.3 \pm 2.3 a b$ \\
\hline 5 & $136.2 \pm 36.4 a b c$ & $143.9 \pm 20.6 a b$ & $59.9 \pm 19.5 a$ & $33.7 \pm 8.5 \mathrm{ab}$ & $0.5 \pm 0.1 \mathrm{a}$ & $0.5 \pm 0.1 \mathrm{ab}$ & $13.6 \pm 3.6 a$ & $14.4 \pm 2.1 \mathrm{ab}$ \\
\hline 6 & $80.1 \pm 26.5 b c$ & $64.9 \pm 32.7 b$ & $34.8 \pm 37.8 a$ & $16.7 \pm 9.0 \mathrm{~b}$ & $0.2 \pm 0.1 \mathrm{c}$ & $0.1 \pm 0.1 c$ & $8.0 \pm 2.7 a$ & $6.5 \pm 3.3 b$ \\
\hline 7 & $146.9 \pm 24.2 \mathrm{a}$ & $188.0 \pm 30.1 \mathrm{a}$ & $53.0 \pm 20.4 \mathrm{a}$ & $46.2 \pm 17.1 \mathrm{a}$ & $0.5 \pm 0.1 a$ & $0.6 \pm 0.1 \mathrm{a}$ & $14.7 \pm 2.4 a$ & $18.8 \pm 3.0 a$ \\
\hline 8 & $129.1 \pm 32.8 \mathrm{abc}$ & $94.5 \pm 62.2 \mathrm{~b}$ & $47.6 \pm 18.2 a$ & $27.7 \pm 14.0 \mathrm{ab}$ & $0.5 \pm 0.1 a$ & $0.3 \pm 0.2 b c$ & $12.9 \pm 3.3 a$ & $9.5 \pm 6.2 b$ \\
\hline 9 & $107.2 \pm 34.9 a b c$ & $147.8 \pm 39.1 \mathrm{ab}$ & $31.9 \pm 8.1 \mathrm{a}$ & $34.9 \pm 8.6 \mathrm{ab}$ & $0.3 \pm 0.1 \mathrm{abc}$ & $0.4 \pm 0.1 \mathrm{ab}$ & $10.7 \pm 3.5 a$ & $14.8 \pm 3.9 \mathrm{ab}$ \\
\hline 10 & $77.8 \pm 22.8 \mathrm{c}$ & $137.2 \pm 13.7 \mathrm{ab}$ & $30.2 \pm 10.3 a$ & $34.4 \pm 7.5 \mathrm{ab}$ & $0.2 \pm 0.1 b c$ & $0.4 \pm 0.1 \mathrm{abc}$ & $7.7 \pm 2.3 a$ & $13.7 \pm 1.4 a b$ \\
\hline Valor de & 0.0041 & 0.0037 & 0.6198 & 0.1488 & $<0.0001$ & 0.0002 & 0.0304 & 0.0037 \\
\hline
\end{tabular}


$T_{2^{\prime}}, T_{3^{\prime}}, T_{4^{\prime}}, T_{5^{\prime}}, T_{8^{\prime}}, T_{9}$ y $T_{10^{\prime}}$ pero diferente de $T_{6}$, que fue el tratamiento con menor número de hongos cosechados por bolsa.

\section{DISCUSIÓN}

\section{Composición bromatológica de los sustratos}

Las mezclas de sustratos (tratamientos) evaluados en el presente estudio, permitieron explorar una variedad de concentraciones de nutrientes disponibles para el cultivo de Pleurotus spp., los cuales incluyeron valores de nitrógeno entre 0.41 y $1.31 \%$, relaciones $\mathrm{C} / \mathrm{N}$ entre 39.79 hasta 131.41 y contenidos de azúcares reductores entre 13.76 a $23.09 \%$ (Tablas 2 y 3 ), entre otros parámetros evaluados.

Los contenidos de carbono, nitrógeno y proteína del olote de maíz y el pasto se encuentran dentro de los intervalos de reportes previos, $51 \%$ para carbono, de 2.5-3.3 \% para proteína y una relación $\mathrm{C} / \mathrm{N}$ de 96.5132.7 (Reyes et al. 2009; Colmenares-Cruz et al. 2017; Morales y Sánchez 2017), a excepción de Hoa et al. (2015) quienes reportaron para el olote de maíz un contenido de nitrógeno y una relación $\mathrm{C} / \mathrm{N}$ diferentes (1.16 y $34.57 \%$, respectivamente). La pulpa de café presentó una menor cantidad de proteína y azúcares reductores y una mayor cantidad de fibra que la reportada comúnmente (Braham y Bressan, 1978), esto probablemente se debe a que la pulpa utilizada en estos experimentos provenía de un beneficio seco de café variedad Robusta (Coffea canephora), la cual lleva un procesamiento postcosecha de secado directo, diferente del tratamiento de despulpado y acumulación en fresco de la pulpa que caracteriza a los granos de la otra especie cultivada, C. arabica. Los subproductos obtenidos por ambos procesos son diferentes, el beneficiado seco proporciona una mezcla de fragmentos de pulpa y cascabillo, como el utilizado en la presente investigación, mientras que con el beneficiado húmedo se obtiene la pulpa entera con residuos del mucílago que acompaña al grano y sin cascabillo. Esto explicaría el menor contenido de azúcares y más fibra en las muestras aquí analizadas.

Por otra parte, el contenido de fibra cruda de los 10 tratamientos evaluados se encuentra dentro de los porcentajes señalados en la literatura (30 al $60 \%$, Koutrotsios et al. 2014). La variación aquí observada entre las diferentes combinaciones de sustrato (38.01 a 39.84 $\%$, Tabla 2) no fue suficiente para demostrar un efecto en la producción de hongos, más bien, indica que los sustratos probados tenían suficiente materia lignocelulósica para el cultivo de hongos de pudrición blanca. El contenido de grasas de los tratamientos evaluados osciló entre 0.2 y $1.04 \%$. La literatura reporta que los sustratos que no sobrepasan contenidos lipídicos de 1 $\%$, favorecen una lenta descomposición (Heredia-Solis et al. 2014). El contenido de cenizas varió de 3.01 a $7.7 \%$ siendo acorde con estudios previos que indican disponibilidad adecuada para los requerimientos minerales en el crecimiento de Pleurotus spp. (Castillo et al. 2012; Heredia-Solis et al. 2014).

\section{Producción y rendimiento}

El sustrato donde se obtuvieron los valores más altos de eficiencia biológica (EB) para ambas cepas fue el pasto pangola, que presentó un contenido de proteína de $3.32 \%$ y nitrógeno de $0.53 \%$ (Tabla 2) y una relación $\mathrm{C} / \mathrm{N}$ de 96.75 (Tabla 3). De acuerdo con la literatura, un alto rendimiento del cultivo de Pleurotus sp. se obtiene a partir de sustratos que contengan nitrógeno entre 0.5-1.2\% con base a materia seca (Dündar y Yildiz 2009). Además, el pasto pangola presentó una composición adecuada de fibra y una alta concentración de azúcares reductores, que, adicionado de una alta concentración de ácidos acético, propiónico y butírico, resulta en una mayor disponibilidad de energía (Reyes et al. 2009). Esto podría explicar por qué este pasto resulta un buen sustrato para Pleurotus spp. y otros hongos comestibles (Barrios-Espinoza et al. 2009; Colmenares-Cruz et al. 2017; Morales y Sánchez 2017).

Llama la atención que los sustratos que produjeron mayor EB no fueron aquellos que contenían más nitrógeno. De hecho, para ambas cepas, el sustrato que produjo valores estadísticamente bajos de EB fue la pulpa de café $\left(T_{6}\right)$, que tenía la concentración más alta de nitrógeno y proteína (1.31 y $8.21 \%$, respectivamente) y una relación $\mathrm{C} / \mathrm{N}$ de 39.79 (Tablas 2 y 3 ). El contenido de nitrógeno y de proteína en el sustrato, junto con el carbono, son nutrientes esenciales para el crecimiento del micelio, la colonización y formación de primordios, así como el desarrollo de cuerpos fructíferos (Sánchez et al. 2008). Esta situación sugiere que las concentraciones de nitrógeno y proteína del sustrato son importantes hasta cierto punto, pero que la producción y la productividad no dependen únicamente de ellas, sino también de otros factores, como por ejemplo: otros 
nutrientes de fácil acceso y de la microbiota presente en la micósfera, la cual influye de manera determinante por la presencia de microorganismos benéficos que estimulan el crecimiento del hongo (Torres-Ruiz et al. 2016), y aún de otros microorganismos con genes que implican capacidad para fijar nitrógeno (Sánchez y Royse 2017). El género Pleurotus spp. en su fase de crecimiento micelial, consume preferentemente carbohidratos solubles y simples (Sharma et al. 2013), en este sentido, se observa que la pulpa de café fue el sustrato con menor contenido de azúcares reductores y carbohidratos entre los tratamientos probados (Tabla 2).

En ambas cepas, se observó que, a mayor porcentaje de pasto en los tratamientos, mayor es la EB, mientras que a mayor porcentaje de pulpa de café se observa un descenso de dichos valores (Figura 2). Por ello, cuando el sustrato fue $100 \%$ de pulpa de café $\left(T_{6}\right)$ se observó el valor más bajo. Esto no concuerda con lo reportado por otros autores, quienes afirman que los residuos de café son un excelente sustrato para el cultivo del género Pleurotus, en particular la pulpa. Incluso, se han reportado valores de EB de hasta 175 \% (Martínez Carrera 1989; García et al. 2006; García-Oduardo et al. 2011). La baja EB podría ser atribuida al tipo de residuos de café que se usó en los sustratos. Mendoza et al. (2019) reportaron un valor de EB de $15.7 \%$, un valor más bajo al obtenido en este trabajo (16.9\%), en $P$. ostreatus sobre los residuos de café $C$. arabica. Por otro lado, Savón et al. (2018) reportaron valores de EB entre 57.7 y 59.4 \% en dos cepas de Pleurotus spp. utilizando como sustrato pulpa de café obtenida de C. canephora. Nieto-Juárez et al. (2019) reportaron diferentes valores de EB del cultivo de $P$. ostreatus sobre pulpa de café provenientes de diferentes lugares, aun tratándose de la misma variedad, por lo que el lugar de procedencia de los sustratos influye sobre su calidad.

El T 1 (100\% olote de maíz), presentó el contenido de nitrógeno más bajo, abajo del valor mínimo de $0.5 \%$ recomendado para el cultivo de hongos (Dündar y Yildiz 2009), por ello, la preparación de mezclas de sustratos favoreció elevar el $0.41 \%$ de nitrógeno y el 0.2 $\%$ inicial de grasas (Tablas 2 y 3 ), aunque la grasa no es un nutriente esencial, puede tener un efecto benéfico en el desarrollo micelial que implique una mejor disponibilidad de energía (Forero et al. 2008; Reyes et al. 2009).
Los valores de EB obtenidos en el presente estudio son bajos en comparación a lo reportado por Barrios-Espinoza et al. (2009), quienes alcanzaron una EB de hasta $112 \%$ en cepas de $P$. ostreatus, cultivado en pasto pangola pasteurizado previamente por composteo en cajones. Por su parte, Torres-Ruiz et al. (2016) aislaron microorganismos promotores en la micósfera de $P$. ostreatus después de la pasteurización del sustrato, los cuales tuvieron efecto positivo en el desarrollo de micelio y crecimiento de basidiomas. En este trabajo, los sustratos fueron esterilizados en autoclave, que, adicionadas a variaciones en el sustrato y la cepa utilizada, pueden explicar las diferencias de productividad reportadas.

En cuanto a la TP y R, los valores obtenidos fueron inferiores a los reportados por Romero et al. en 2010 (TP de $2.2 \%$ utilizando de hojas de plátano para el cultivo de $P$. ostreatus), mientras que Sánchez et al. (2008) reportaron una TP de $2.9 \%$ para cultivo de $P$. ostreatus en rastrojo de tomate. Los valores de rendimiento para la cepa ECS-123 variaron entre 7.7-14.7\% y no se presentaron diferencias significativas entre los tratamientos, mientras que en la cepa ECS-1123 estos valores fluctuaron entre 6.5 hasta $18.8 \%$. En general, en la primera cosecha se obtuvo entre 60 y $70 \%$ de la producción de basidiomas totales, que coincide con lo reportado por Pérez Merlo y Mata (2005) y Gaitán-Hernández et al. (2006).

Un parámetro físico importante de resaltar es la compactación del sustrato. Se observó que las bolsas de $\mathrm{T}_{6}$ (100\% pulpa de café), estaban más compactas con respecto a otros tratamientos que se veían más esponjados o voluminosos. Algunos autores reportan que la compactación del material afecta el intercambio de gases, favoreciendo el aumento de la concentración de $\mathrm{CO}_{2}$, disminuyendo el $\mathrm{O}_{2}$ disponible y provocando condiciones adversas para el rendimiento del hongo (López-Rodríguez et al. 2008; Liang et al. 2009); por ello, se considera que cuando la pulpa de café estuvo mezclada con otros sustratos, ya sea pasto, olote o ambos, se alcanzaron mejores resultados de productividad. García-Oduardo et al. (2011) afirman que la pulpa café debe ser mezclada o suplementada con otros subproductos agrícolas (pajas de cebada y trigo, bagazo de caña de azúcar y rastrojo de maíz) para favorecer la fermentación aerobia de la misma y ofrecer mejores valores de EB, TP y R. 


\section{CONCLUSIONES}

De los 10 tratamientos analizados, en el tratamiento $7\left(T_{7}=100 \%\right.$ pasto pangola) se obtuvieron los valores más altos de EB, TP y $R$ con las cepas utilizadas, aunque, estos valores no fueron estadísticamente significativos de los tratamientos que contenían pasto en sus formulaciones $\left(T_{1}, T_{2}, T_{3}, T_{4}, T_{5}, T_{9}\right)$. Los resultados del presente estudio confirman que una concentración de nitrógeno de 0.5 \% y una relación $\mathrm{C} / \mathrm{N}$ de 96.75 son suficientes para obtener una buena producción de basidiomas, además del contenido de azúcares reductores y grasas simples en el sustrato.

\section{AGRADECIMIENTOS}

Los autores agradecen a la M. C. Lilia Moreno Ruíz por sus aportes técnicos y al M. C. Javier Valle Mora por su ayuda en el análisis estadístico de los datos. Este estudio fue financiado con recursos fiscales de El Colegio de la Frontera Sur (ECOSUR) de México y con fondos del proyecto CONACYT No. 9342634372. Así también, el primer autor agradece a La Secretaría del Trabajo y Previsión Social (STPS) del programa Jóvenes Construyendo el Futuro por la beca otorgada durante un año, lo cual permitió participar en esta investigación como parte de capacitación para el trabajo.

\section{LITERATURA CITADA}

Abid AH, Hamid A, Naz RMM, Shah SZA, Anjum S, Khan MT, llyas M. 2020. Impact of different lignocellulose substrates on growth and yield of oyster mushroom (Pleurotus ostreatus). Pure and Applied Biology (PAB) 9, 768-775. http://dx.doi.org/10.19045/ bspab.2020.90083

Adams RC, MacLean FS, Dixon JK, Bennett FM, Martin Gl, Lough RC. 1951. The utilization of organic wastes in N.Z. (concid.) second interim report of the inter-departmental committee. Engineering, New Zealand. 396-424.

Barrios-Espinoza BM, Moreno-Ruiz L, Sánchez JE. 2009. Composteo en cajones de madera como pretratamiento del sustrato para cultivar Pleurotus ostreatus. Revista Mexicana de Micología 29, 51-59.

Bitew TD, Mandefro SA. 2018. Substrate optimization for cultivation of Pleurotus ostreatus on lignocellulosic wastes (coffee, sawdust, and sugarcane bagasse) in Mizan-Tepi University, Tepi Campus, Tepi Town. Journal of Applied Biology and Biotechnology 6, 1420. https://doi.org/10.7324/JABB.2018.60403

Braham JE, Bressani R. 1978. Pulpa de café. Composición, tecnología y utilización. Instituto de Centro América y Panamá. Escala, Bogotá.

Castillo F, Rodríguez R, Prieto F, Román A. 2012. Caracterización física y química proximal de paja, grano y almidón de cebada de la variedad Esmeralda. BioTecnología 16, 9-20. https://doi. org/10.1016/j.ibiod.2008.12.006
Chang ST, Wasser S. 2018. Current and future research trends in agricultural and biomedical applications of medicinal mushrooms and mushroom products. International Journal of Medicinal Mushrooms 20, 1121-1133. https://doi.org/10.1615/IntJMedMushrooms. 2018029378

Colmenares-Cruz S, Sánchez JE, Valle-Mora J. 2017. Agaricus bisporus production on substrates pasteurized by self-heating. AMB Express 7, 135-144. https://doi.org/10.1186/s1356801704386 Cruz-Ornelas R, Sánchez JE, Amaya-Delgado L, Guillén-Navarro K, Calixto-Romo A. 2019. Biodegradation of NSAIDs and their effect on the activity of ligninolytic enzymes from Pleurotus djamor. 3 Biotech 9, 373-381. https://doi.org/10.1007/s13205-0191904-4

Del Pilar Ríos M, Hoyos JL, Sánchez SA. 2010. Evaluación de los parámetros productivos de la semilla de Pleurotus ostreatus propagada en diferentes medios de cultivo. Biotecnología en el Sector Agropecuario y Agroindustrial 8, 86-94.

Dündar A, Yildiz A. 2009. A comparative study on Pleurotus ostreatus (Jacq.) P.Kumm. cultivated on different agricultural lignocellulosic wastes. Turkish Journal Biology 33, 171-179. https://doi. org/10.3906/biy-0804-2

FAO (Food and Agriculture Organization of the United Nations). 2017. Disponible en: http://www.fao.org/faostat/es/\#data/QC (20 de marzo, 2020).

Forero CL, Hoyos OL, Bazante WE. 2008. Evaluación de residuos de ají (Capsicum spp.) como sustrato en la producción de setas comestibles (Pleurotus ostreatus). Facultad de Ciencias Agropecuarias 6, 42-53.

Gaitán-Hernández R, Esqueda M, Gutiérrez A, Sánchez A, Beltrán-García M, Mata G. 2006. Bioconversion of agrowastes by Lentinula edodes: the high potential of viticulture residues. Applied Microbiology and Biotechnology 71, 432-439. https://doi. org/10.1007/s00253-005-0241-1

Gaitán-Hernández R, Silva-Huerta A. 2016. Aprovechamiento de residuos agrícolas locales para la producción de Pleurotus spp., en una comunidad rural de Veracruz, México. Revista Mexicana de Micología 43, 43-47.

García N, Bermúdez R, Gross P, Hernández M. 2006. Cultivo de cepas de Pleurotus spp. sobre pulpa de café. Revista Mexicana de Micología 3, 99-101.

García-Oduardo N, Bermúdez-Savón RC, Serrano-Alberni M. 2011. Formulaciones de sustratos en la producción de setas comestibles Pleurotus. Tecnología Química 31, 272-282.

Girmay Z, Gorems W, Birhanu G, Zewdie S. 2016. Growth and yield performance of Pleurotus ostreatus (Jacq. Fr.) Kumm (oyster mushroom) on different substrates. AMB Express 6, 87-94. https://doi.org/10.1186/s13568-016-0265-1

Gupta S, Summuna B, Gupta M, Annepu SK. 2018. Edible mushrooms: cultivation, bioactive molecules, and health benefits. In: Mérillon J.M., Ramawat K.G. (eds.), Bioactive molecules in food, reference series in phytochemistry. Springer, Berlin. 1-33.

Heredia-Solis A, Esparza-lbarra EL, Romero-Bautista L, Cabral-Arellano F, Bañuelos-Valenzuela R. 2014. Bagazos de Agave salmiana y Agave weberi utilizados como sustrato para producir Pleuro- 
tus ostreatus. Revista Iberoamericana de Ciencias 5, 102-108. https://doi.org/10.18779/cyt.v10i2.164

Hoa HT, Wang CL, Wang CH. 2015. The effects of different substrates on the growth, yield, and nutritional composition of two oyster mushrooms (Pleurotus ostreatus and Pleurotus cystidiosus). Mycobiology 43, 423-434. https://doi.org/10.5941/ MYCO.2015.43.4.423

Koutrotsios G, Mountzouris KC, Chatzipavlidis I, Zervakis GI. 2014. Bioconversion of lignocellulosic residues by Agrocybe cylindracea and Pleurotus ostreatus mushroom fungi-Assessment of their effect on the final product and spent substrate properties. Food chemistry 161, 127-135. https://doi.org/10.1016/j.foodchem.2014.03.121

Liang ZC, Wu CY, Shieh ZL, Cheng SL. 2009. Utilization of grass plants for cultivation of Pleurotus citrinopileatus. International Biodeterioration and Biodegradation 63, 509-514, https://doi. org/10.1016/j.ibiod.2008.12.006

López-Rodríguez C, Hernández-Corredor R, Suárez-Franco C, Borrero M. 2008. Evaluación del crecimiento y producción de Pleurotus ostreatus sobre diferentes residuos agroindustriales del departamento de Cundinamarca. Universitas Scientiarum 13, 128-137.

Martínez-Carrera D. 1989. Simple technology to cultivate Pleurotus on coffee pulp in the tropics. Mushroom Science 12, 169-178.

Mendoza H, Juscamaita J, Lawrence Q. 2019. Análisis de la producción del hongo comestible Pleurotus ostreatus obtenida a partir de los subproductos de la etapa de despulpado del café. Agroindustrial Science 9, 179-187. http://dx.doi.org/10.17268/ agroind.sci.2019.02.11

Morales DV, Sánchez JE. 2017. Self-heating pasteurization of substrates for culinary-medicinal mushrooms cultivation in Mexico. International Journal of Medicinal Mushrooms 19, 477-484. https://doi.org/10.1615/IntJMedMushrooms.v19.i5.90

Moreno-Ruiz L, Andrade RH, Sánchez-Vázquez JE. 2014. Biotechnological potential of ten Pleurotus djamor strains. In: Proceedings of 8th International Conference on Mushroom Biology and Mushroom Products. Directorate of Mushroom Research, New Delhi. 262-270.

Motato KE, Mejía Al. 2006. Evaluación de los residuos agroindustriales de plátano (Musa paradisiaca) y aserrín de abarco (Cariniana piriformes) como sustratos para el cultivo del hongo Pleurotus djamor. Vitae 13, 24-29.

Nieto-Juárez JI, Ruiz AD, López WA. 2019. Estudio preliminar de la composición nutricional del hongo Pleurotus ostreatus cultivado en pulpa de café. Revista de la Sociedad Química del Perú 85, 422-431. http://dx.doi.org/10.37761/rsqp.v85i4.256

Pérez Merlo R, Mata G. 2005. Cultivo y selección de cepas de Pleurotus ostreatus y $P$. pulmonarius en viruta de pino: obtención de nuevas cepas y evaluación de su producción. Revista Mexicana de Micología 20, 61-70.

Periasamy K, Natarajan K. 2004. Role of lignocellulosic enzymes during basidiomata production by Pleurotus djamor variety roses. Indian Journal of Biotechnology 3, 577-583.

Piña-Guzmán AB, Nieto-Monteros DA, Robles-Martínez F. 2017. Utilización de residuos agrícolas y agroindustriales en el cultivo y producción del hongo comestible seta (Pleurotus spp.). Revista Internacional de Contaminación Ambiental 32, 141-151. https:// doi.org/10.20937/RICA.2016.32.05.10

Quimio TH. 2002. Tropical mushroom cultivation. National Book Store, Mandaluyong.

Reyes A, Soto M, Ornelas E, Treviño E, Negrete J, Barragán H. 2009. Estimación del valor nutricional de pastos tropicales a partir de análisis convencionales y de la producción de gas in vitro. Técnica Pecuaria en México 47, 55-67. https://doi.org/10.22319/ RMCP.V47I1.1488

Romero O, Huerta M, Damián MA, Macías A, Tapia AM, Parraguirre JF, Juárez J. 2010. Evaluación de la capacidad productiva de Pleurotus ostreatus con el uso de hoja de plátano (Musa paradisiaca I., cv. Roatan) deshidratada, en relación con otros sustratos agrícolas. Agronomía Costarricense 34, 53-63.

Romero-Arenas O, Ita VD, Ángeles M, Rivera-Tapia JA, Tello-Salgado I, Espino-Barros OA, Damián-Huato M.A. 2018. Capacidad productiva de Pleurotus ostreatus utilizando alfalfa deshidratada como suplemento en diferentes sustratos agrícolas. Agricultura, Sociedad y Desarrollo 15, 145-160. https://doi.org/10.22231/ asyd.v15i2.788

Royse DJ, Baars J, Tan O. 2017. Current overview of mushroom production in the world. In: Zied D.C., Pardo-Giménez A. (eds.), Edible and medicinal mushrooms: technology and applications. Wiley, Hoboken. 5-13. https://doi.org/10.1002/9781119149446. ch2

Ruan-Soto F. 2018. Recolección de hongos comestibles silvestres y estrategias para el reconocimiento de especies tóxicas entre los tsotsiles de Chamula, Chiapas, México. Scientia Fungorum 48, 1-13. https://doi.org/10.33885/sf.2018.48.1179

Sánchez A, Esqueda M, Gaitán-Hernández R, Córdova A, Coronado ML. 2008. Uso potencial del rastrojo de tomate como sustrato para el cultivo de Pleurotus spp. Revista Mexicana de Micología $28,17-24$.

Sánchez JE, Royse DJ. 2017. La biología, el cultivo y las propiedades nutricionales y medicinales de las setas Pleurotus spp. Microorganismos benéficos asociados a la micósfera de Pleurotus spp. Uteha-Ecosur, San Cristóbal de las Casas. 53-61.

SAS Institute, 2007. JMP. SAS Institute Statistical Analysis System, Cary.

Savón RC, Oduardo NG, Agbozouhoue KK, Alberni MS. 2018. Evaluación de la productividad de dos cepas de Pleurotus spp. sobre pulpa de café Coffea canephora pierre ex frhoener. Tecnología Química 38, 284-293. https://doi.org/10.1590/22246185.2018.2.\%x

Sharma S, Yadav RK, Pokhrel CP. 2013. Growth and yield of oyster mushroom (Pleurotus ostreatus) on different substrates. Journal on New Biological Reports 2, 3-8. https://doi.org/10.1186/ s13568-016-0265-1

Torres-Ruiz E, Sánchez JE, Guillén-Navarro GK, Ramos-Pérez DG, Royse DJ. 2016. Microbial promoters of mycelial growth, fruiting and production of Pleurotus ostreatus. Sydowia 68, 151-161. https://doi.org/10.12905/0380.sydowia68-2016-0151 
Tshinyangu KK, Hennebert GL. 1995. Effects of synthetic nutrient carriers on the fruiting of Pleurotus ostreatus variety columbinus. Bioresource Technology 54, 249-254.

Wani BA, Bodha RH, Wani A.H. 2010. Nutritional and medicinal importance of mushrooms. Journal of Medicinal Plants Research 4, 2598-2604. https://doi.org/10.5897/JMPR09.565
Williams S, 1984. Official methods of analysis. Association of Official Analytical Chemists, Inc., Arlington. 